Military Technical College

Kobry El-Kobbah, Cairo, Egypt

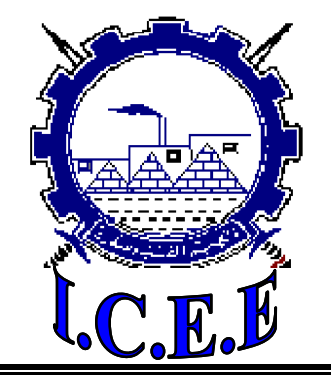

CT-3 $5^{\text {th }}$ International Conference on

Chemical \& Environmental

Engineering

25 - 27 May, 2010.

\title{
USING ELECTRON BEAM FOR REMOVING SOME METAL IONS FROM WASTEWATER BY HUMIC ACID
}

\author{
Naima A. El-Gendy ${ }^{*}$ and Abdel-MohtyA.**
}

\begin{abstract}
Electron beam irradiation is an important technique for removing heavy metal ions from their solutions especially of high concentrations. Humic acid is used as organic scavengers $\mathrm{HO}^{\bullet}$ radical to prevent metals from reoxidation and reduce the energy used. The adsorption experiments were carried out on model solutions containing two metals: $\mathrm{Cr}^{6+}$ and $\mathrm{Cs}^{+}$. The removal technique was carried out by irradiation and adsorption using humic acid. The adsorption technique was affected by several factors such as equilibration time, solution $\mathrm{pH}$, conc. of chromium ions and humic acid. Different irradiation doses $25-150 \mathrm{KGy}$, and different concentrations of $\mathrm{Cr}^{6+}$ and $\mathrm{Co}^{2+}$ ions were used. The removal percentage was almost $100 \%$ in the presence of humic acid during irradiation with low dose $25 \mathrm{kGy}$.
\end{abstract}

\section{Keywords}

Electron Beam / Metal Removal / Humic Acid / Adsorption.

\footnotetext{
* Department of Polymer, National Center for Radiation Research and Technology, Egypt

${ }^{* *}$ Hot Laboratories Center, Atomic Energy Authority, Egypt.
} 


\section{Introduction}

Lubrication is simply the use of a material to improve the smoothness of movement of one surface over another, and the materials which are used in this way are called lubricant. Lubricants may be liquid, solid, or gas. Most modern lubricants are petroleum based although vegetable oils and fats are also used. The most important single property of lubricating oil is its viscosity. Lubricating oils are classified as refined and synthetic. Refined oils are produced from crude oil refinery. Crude oil refinery produces paraffinic oils, naphthenic oils and small amount of aromatic oils. Synthetic lubricants are produced via chemical synthesis. Selected chemicals could be blended with the lubricating oils to impart them certain specific properties. Resistance of a lubricant to viscosity change with temperature is determined by its viscosity index (VI) which is an arbitrary number calculated from the observed viscosities at two widely separated temperatures. The normal range of viscosity index is from zero up to 100. Oils of high VI could resist excessive thinning at high temperatures where as those of low VI experience an extremely thinning at high temperatures. Viscosity index of the paraffinic based lubricants is oftenly greater than that of the naphthenic based ones. Viscosity index improvers VII can be regarded as the key to high performance multigrade oil. They are generally oil soluble polymers. The added polymeric molecules may interact with the base oil affecting its final viscosity. Oils containing viscosity index improvers can achive a viscosity index up to 150 .

Three major families of viscosity index improvers are known; olefin copolymers, hydrogenated diene copolymers, and acrylic acid based copolymers [1, 2]. Different polymer concentrations are always tested in order to determine the performance of the additives under the engine running conditions. The optimum improver means higher viscosity index and shear stability. It is also soluble in the base oil and compatible with the other additives.

Schiff et al [3] used hydrogenated random butadiene-styrene copolymer to produce a shear stable, lubricant with viscosity index of about 140 . The butadiene content was ranged from 30 to $44 \%$ weight, and its molecular weight started from 25000 to 125000 . F. Dawans, et al. [5] presented a viscosity index improver based only on the butadiene polymer. Molecular weighs up to about 450000 were employed later on by Forbes and Meldrum [5]. They described a graft copolymer of polystyrene and poly isobutylene. Solubility has an important effect on the viscosity index, as mentioned by Pierre Bataille et al [6]. They prepared and tested two copolymers, $\alpha$-methyl styrene and 2-ethylhexyl acrylate. The copolymers obtained were blended with a paraffinic (SAE 10) of $110 \mathrm{VI}$ and a naphthenic base oil of $56 \mathrm{VI}$. The copolymers were tested according to the ASTM 445 IP-71 method.

Amal M. Nassar et al [7] synthesized polymeric acrylates VI improvers by copolymerization of four esters (decyl acrylate, dodecyl acrylate, tetradecyl acrylate, and hexadecyl acrylate) with different amount of styrene. Their effectiveness as VI improvers was found proportional to molecular weight of the copolymer. Solubility of the prepared copolymers was found inversely proportional to their molecular weight. Rabab Mohamed Elsayed [8] employed four different esters and carried out free radical copolymerization using different monomers (vinyl acetate, maleic anhydride, 1-octene, and 1-tetradecene). The copolymers prepared using vinyl acetate, or octene were found, relatively, more efficient as VI improvers. Effectiveness was found proportional to the alkyl chain length. Those prepared from tetradecene with different alcohols were found to be more effective as pour point depressant than those prepared from dodecene or octane. Effecftiveness as pour point depressants was also found proportional to concentration of the additives and also to chain length of the alkyl groups. Maleate 
copolymers proved to be efficient as viscosity index improvers according to Nehl S. Ahmed [9].

In this work a group of copolymers based on polyacrylates were prepared and evaluated as viscosity index improvers. Polyacrylates have been chosen since they are simply prepared compared with other viscosity index improvers, compatible with other additives, of high shear stability, effective as pour point depressants and of relatively low cost.

\section{Experimental}

\section{Copolymers preparation and characterization: \\ Materials:}

In this work Acrylic acid $\left[\mathrm{H}_{2} \mathrm{C}=\mathrm{CH}-\mathrm{COOH}\right]$ was estrified using a number of alcohols, Octyl

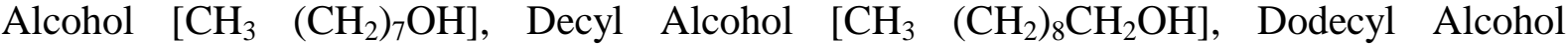
(Dodecanol) $\left[\mathrm{CH}_{3}-\left(\mathrm{CH}_{2}\right)_{11}-\mathrm{OH}\right]$, Tetradecyl alcohol (Tetradecanol) $\left[\mathrm{CH}_{3}-\left(\mathrm{CH}_{2}\right)_{13}-\mathrm{OH}\right]$, Hexadecyl alcohol (Hexadecanol) $\left[\mathrm{CH}_{3}-\left(\mathrm{CH}_{2}\right)_{15}-\mathrm{OH}\right]$. Benzoyl peroxide $\left[\left(\mathrm{C}_{6} \mathrm{H}_{5} \mathrm{CO}\right)_{2} \mathrm{O}_{2}\right]$ was used as an initiator. Hydroquinone (1, 4-Dihydroxybenzene; p-Dihydroxybenzene; or 1,4Benzenediol) $\left[\mathrm{C}_{6} \mathrm{H}_{4}(\mathrm{OH})_{2}\right]$, Catalysts: P-Toluene Sulphonic Acid (PTSA) monohydrate $\left[\mathrm{CH}_{3} \mathrm{C}_{6} \mathrm{H}_{4} \mathrm{SO}_{3} \mathrm{H} \cdot \mathrm{H}_{2} \mathrm{O}\right]$ were used as inhibitor. Solvents have been selected and used according to the process, materials, and reaction conditions (Table 1). Free radical initiators are used to initiate the polymerization process. The initiators were either oxygen-based or nitrogen-based thermally unstable compounds. Their decomposition could yield two free radicals [10]. Acrylic acid is very active, it can undergo polymerization even in absence of any initiators, hence; inhibitors are needed to inhibit its polymerization. After estrification, the esters need to be purified. sodium hydroxide $(\mathrm{NaOH})$ was used to remove the unreacted acid. Anhydrous Calcium chloride $\left(\mathrm{CaCl}_{2}\right)$ and anhydrous Sodium sulphate $\left(\mathrm{Na}_{2} \mathrm{SO}_{4}\right)$ were used to dry the final product. All materials are from Aldrich Chemical Co.Ltd. (U.K).

\section{Procedures:}

Polyacrylates viscosity index improvers have been prepared in several steps, starting from esterification of acrylic acid and alcohols, purification of produced esters, identification of the purified esters, polymerization of the esters, and finally characterization of the produced polymers via solubility test and molecular weight determination.

\section{Estrification of Acrylic Acid with Different Types of Alcohols :}

Esterification has been carried out by reacting one mole of acrylic acid with one mole of the selected alcohol (octyl, decyl, dodecyl, tetradecyl, and hexadecyl). The reactions were carried out in a resin kittle in presence of $0.5 \%$ p-toluene sulphonic acid as a catalyst, $0.25 \%$ hydroquinone as inhibitor, for the polymerization of acrylic acid, and xylene as a solvent. Esterification reactions were carried out under a slow stream of deoxygenated nitrogen and were agitated using a mechanical stirrer at $500 \mathrm{rpm}$. The reactants which were mixed with an equal weight of xylene were heated gradually from the room temperature up to $130^{\circ} \mathrm{C} \pm 5^{\circ} \mathrm{C}$ [11]. The extent of reaction was followed by monitoring the amount of librated water.

\section{Purification of the prepared Esters:}

A suitable amount of charcoal were added to the esters and allowed to reflux for four hours and then filtered. The filtrate was taken and washed by $0.5 \mathrm{~N}$ sodium hydroxide in separating funnel and shake well. The entire process was repeated several times to ensure complete removal of the unreacted acid. The purified ester was then washed several times with distilled 
water to remove any traces of sodium hydroxide then the ester was left overnight on calcium chloride anhydrous for drying. It was then removed by filtration, xylene was removed by distillation. The ester was then ready to use in copolymerization process.

\section{Identification of the purified esters:}

The infrared spectra obtained using an F.T.I.R [Fourier Transform Infra Red] spectrometer model type Mattson-Infinity Series Bench top 961 have been employed for identification of the purified esters before going ahead for copolymerizing them.

\section{Copolymerization of the produced esters:}

Polymeric additives were prepared by free radical copolymerization of equimolar quantities of the prepared esters. The reaction was carried out in a four-necked round bottom flask equipped with a stirrer, efficient condenser, thermometer, and an inlet for introduction of dry nitrogen. Drying of nitrogen took place by passing it through a silica gel bed. The process was carried out by adding in the flask one mole of each of the selected esters together with the desired weight of initiator (benzoyl peroxide). The mixture was heated to $60^{\circ} \mathrm{C} \pm 5^{\circ} \mathrm{C}$ for eight hours in the presence of toluene as a solvent. At the end of the reaction, the temperature was allowed to reduce to the room temperature, and the reaction mixture was then poured drop by drop in cooled methanol with continuous stirring. The mixture was filtered off and dried. Constituents of the prepared polymers and their designation are shown in Table 2.

\section{Characterization of the Prepared Copolymers:}

Solubility of the prepared copolymers in lube oil was investigated before testing their effectiveness as viscosity index improvers. The solubility test took place by dissolving one gram of the polymer in hundred grams of additive free base oil (SAE 30). The (1g / $100 \mathrm{~g}$ ) mixture has been allowed to stand overnight. This time was enough to produce the required swelling for the polymer. The mixture was agitated by a Teflon covered magnetic stirrer for 20 minutes at $60^{\circ} \mathrm{C}$ to disintegrate the gel. To test homogeneity and thermodynamic stability of the mixture, viscosity of five different samples from each solution was measured. Molecular weight of the copolymers was determined by Gel Permeation Chromatography (GPC) model waters 600E.

\section{Viscosity Measurement}

Kinematic viscosity of the oil with and without the tested polymers was determined at $40{ }^{\circ} \mathrm{C}$ and also at $100^{\circ} \mathrm{C}$. Different polymer concentrations $(0.5,1,1.5,2,2.5$, and $3 \%$ by weight $)$ were used to study the effect of polymer concentration on VI of the oil. Measurements took place using a two Cannon-Fenske viscometers, size 300, and 150. This viscometer is good for Newtonian or nearly Newtonian fluids. It is also known as Cannon-Fenske Capillary tube. Each sample was measured three times in order to minimize the error. Finally, the viscosity index was determined according to the ASTM D-2270-87 formula

$\mathrm{VI}=[(\mathrm{L}-\mathrm{U}) /(\mathrm{L}-\mathrm{H})] \times 100$

Values of $\mathrm{L}$ and $\mathrm{H}$ were obtained from the tables of ASTM D-2270-87 where the basic kinematic viscosity at $100{ }^{\circ} \mathrm{C}$ are equal to or more than $2 \mathrm{cSt}$ and less than $70 \mathrm{cSt}$. When the basic kinematic viscosity at $100{ }^{\circ} \mathrm{C}$ is above $70 \mathrm{cSt}$, the values of $\mathrm{L}$ and $\mathrm{H}$ are to be calculated as follows: 
$\mathrm{L}=0.8353 \mathrm{Y} 2+14.67 \mathrm{Y}-216$

$\mathrm{H}=0.1684 \mathrm{Y} 2+11.85 \mathrm{Y}-97$

If the measured viscosity at $100^{\circ} \mathrm{C}$ is not listed in the tables, but within the range of $2-70 \mathrm{cSt}$, the corresponding values of $\mathrm{L}$ and $\mathrm{H}$ can be obtained by linear interpolation. When the calculated VI is above 100, it has to be recalculated, as follows

$$
\begin{aligned}
& \mathrm{YN}=\mathrm{H} / \mathrm{U} \\
& \mathrm{N}=(\log \mathrm{H}-\log \mathrm{U}) / \log \mathrm{Y} \\
& \mathrm{VI}=\{[(\operatorname{anti} \log \mathrm{N})-1] / 0.00715\}+100
\end{aligned}
$$

\section{Results and Discussion}

According to the I.R spectrum shown in Figure (4), the esters afford similar I.R spectrum and estrification is nearly completed. Solubility tests proved the formation of true solutions and showed that the copolymers could be dissolved molecularly in the base oil.

\section{Effect of the prepared copolymers as viscosity index improvers}

Kinematic viscosity of the oil containing different concentration of the tested additives was determined at $40^{\circ} \mathrm{C}$ and $100^{\circ} \mathrm{C}$. The results are given in Tables 3 and 4 .

Thickening effect is noticeable at both temperatures however; this effect is much greater at $100{ }^{\circ} \mathrm{C}$ (up to $175 \%$ ) than that at $40{ }^{\circ} \mathrm{C}$ (up to $152 \%$ ). Thickening is a desirable behavior so that a little contribution is observed at low temperature and a higher one is expected at high temperatures. The parameters which may have a noticeable effect on the effectiveness are molecular weight, alkyl chain length, and concentration.

\section{Effect of the additive concentration on the viscosity index}

Six different concentrations from 0 to $3 \%$ by weight have been prepared from each additive to study the effect of concentration on the additive efficiency as viscosity index improver. The results are given in Table 5 indicate that the viscosity index increases monotonically with increasing the concentration of the additive. As the temperature increases, the oil viscosity decreases, meanwhile the polymer molecule expands due to the increase in the solvation power and consequently the size of micelle increases. This increase in the micelle size is schematically illustrated in Figure (5). It counterbalances the reduction in the lube oil viscosity and, hence, decreases the change in the viscosity with the temperature. The process of coil expansion is entirely reversible. Coil contraction occurs with decreasing the oil temperature [10]. Increasing the additive concentration makes further increase in number of coils and higher viscosity index is therefore expected.

\section{Effect of molecular weight on the viscosity index}

Molecular weights of the prepared copolymers have been measured and the results are given in Table 6. It has been noticed that the molecular weight of polymers is proportional to the alkyl chain length. It is generally known that the thickening properties of the viscosity index improver are directly related to the immensely greater molecular size compared to that of the base oil in which it is dissolved [10]. In the lattice theory of viscous flow, segment of polymer molecules fills holes in the lattice (constructed of all surrounding molecules) and thereby limit the ability of smaller molecules to participate in movement through the lattice. Consequently, 
as the molecular weight increases, the hydrodynamic size increases, and that influence thickening at all temperatures. Figure 7 illustrates such effect where the viscosity index increases by increasing molecular weight of the additives.

\section{Effect of alkyl chain length on the viscosity index}

Figure 8 shows the effect of the alkyl chain length on effectiveness of the tested viscosity index improvers .It has been noticed that this effectiveness is directly proportional to the alkyl chain length. This may also be attributed to the micelle size which is expected to increase by increasing lengths of the alkyl chains.

\section{Conclusions}

1. The prepared compounds would be effective as viscosity index improvers, and their effectiveness could be increased by increasing either the molecular weight of copolymer from (140,000 up to 236,000 ) or the alkyl chain length from (8 up to 16). For the copolymer A, B, $\mathrm{C}$ and D; the effect of both molecular weight and the chain length are obvious. When either the molecular weight or the chain length increases, the viscosity index increases. It also means formation of effective coiled copolymers. For the copolymers $\mathrm{E}$ and $\mathrm{F}$ which are characterized by low molecular weights, the viscosity index is relatively low, the chain length is not highly effective. Thses results means that the viscosity index depends mainly on the molecular weight of the copolymer and the role of chain length appears only with molecular weights exceeding 200000.

2. Effectiveness of the prepared additives as viscosity index improvers increases by increasing their concentrations in the base oil. There are no reflection points through the tested range of concentrations and a concentration of $3 \%$ by wt. offers a viscosity index ranged from 120 up to 143 according to both the molecular weight and the alkyl chain length.

\section{References}

[1] J. George Wills, "Lubrication Fundamentals", Marcel Dekker, 1980

[2] A. I. Akhmedov and D. A. Mamedova. "4-methyl-1-Pentene Olegomers as Thickening Agents", Chemistry and Technology of Fuels and Oils, Vol. 39, No.6, 2003, pp.339-342

[3] Sidney Schiff, Marvin M. Johnson, and William L. Streets; US patent, 3,554,911 (1971).

[4] Dawans, Francois, Goldenberg, Emmanuel, Durand, and Jean-Pierre, "Lubricating Oil Compositions Containing Hydrogenated Polybutadiene Viscosity Index Improvers", US patent no 3959161, (1993)

[5] E. S. Forbes, Lightwater, I. G. Meldrum; US Patent 3,992,310 (1976)

[6] Pierre Bataille, Naser Sharifi-Sanjani, and Esamail Evin, "Preparation and Characterization of a Viscosity Index Improver for Naphthenic and Paraffinic Base Oils", journal of solution chemistry, vol. 23, No. 2, 1994, 325-337

[7] Amal M. Nassar, Nehal S. Ahmed, Rasha S. Kamal, and Abdel-Azim A. Abdel Azim, and E. I. El-Nagdy, "Preparation and Evaluation of Acrylate Polymers as Viscosity Index Improvers for Lube Oil", Petroleum Science and Technology, 23, 2005, PP 537-546

[8] Rabab M. Elsayed, "Synthesis and Evaluation of some Lubricating oil Viscosity Index Impovers and Pour Point Depressants", M.Sc Thesis, University of Menoufia, Egypt, 2006 
[9] Nehal S. Ahmed, "Lubricants Additives from Maleate copolymers", Petroleum Science and Technology, 26:3, 2008, PP 298-306,

[10] Leslie R. Rudnick, "Lubricant Additives; chemistry and applications", Marcel Dekker, Inc., 2003, PP 329-353

[11] ZA Sadykhov, T.G. Khanlarov, N.A. Nechitailo, M.A. Dzyubina, and P.I. Sanin, "Thermal Stability of Copolymers of Isobutylene With Certain $\alpha$-Methylstyrene Derivatives", Khimiya I Tekhnologiya Topliv I Masel, No. 6, 1974, pp. 9-12,
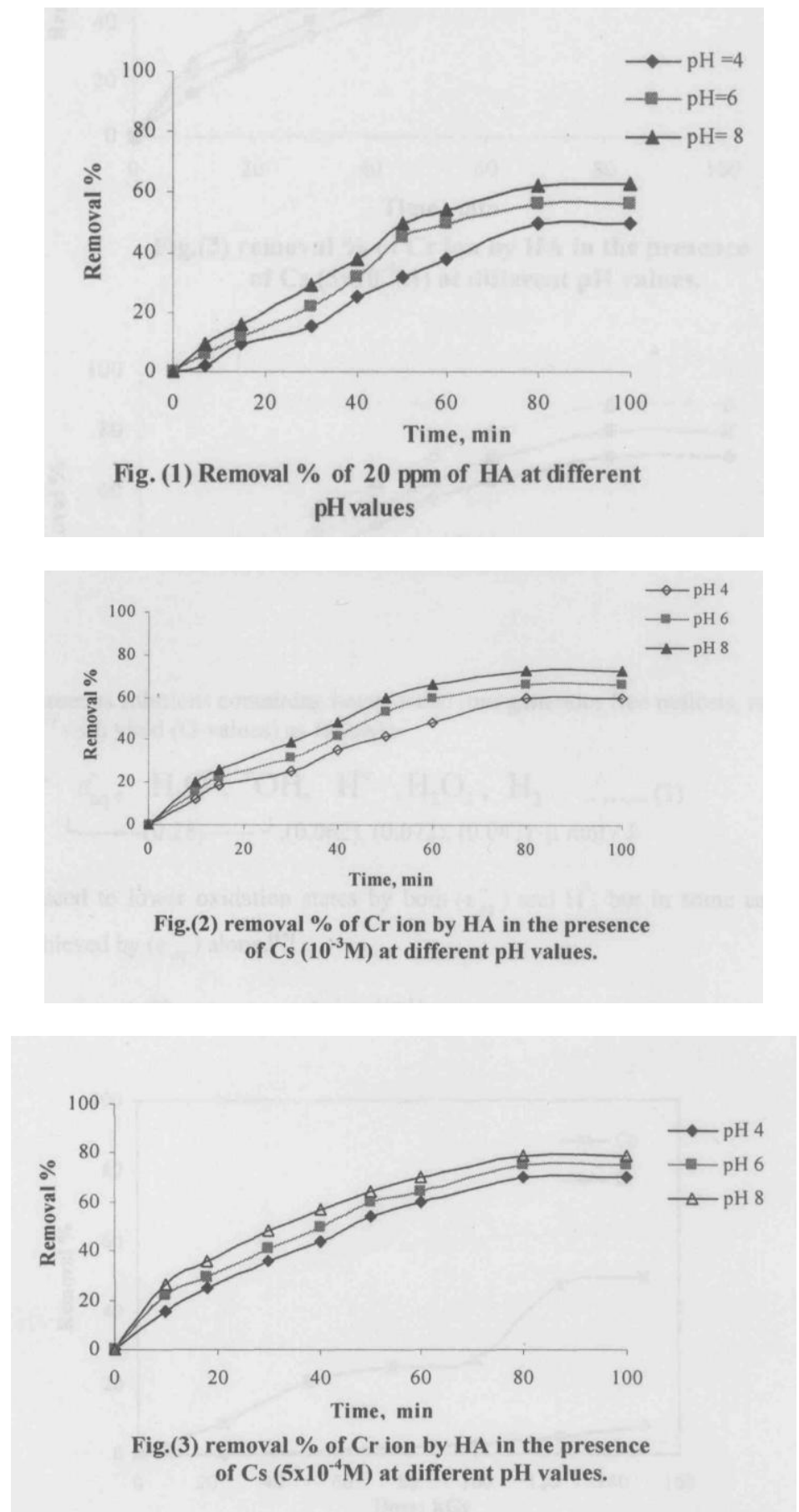

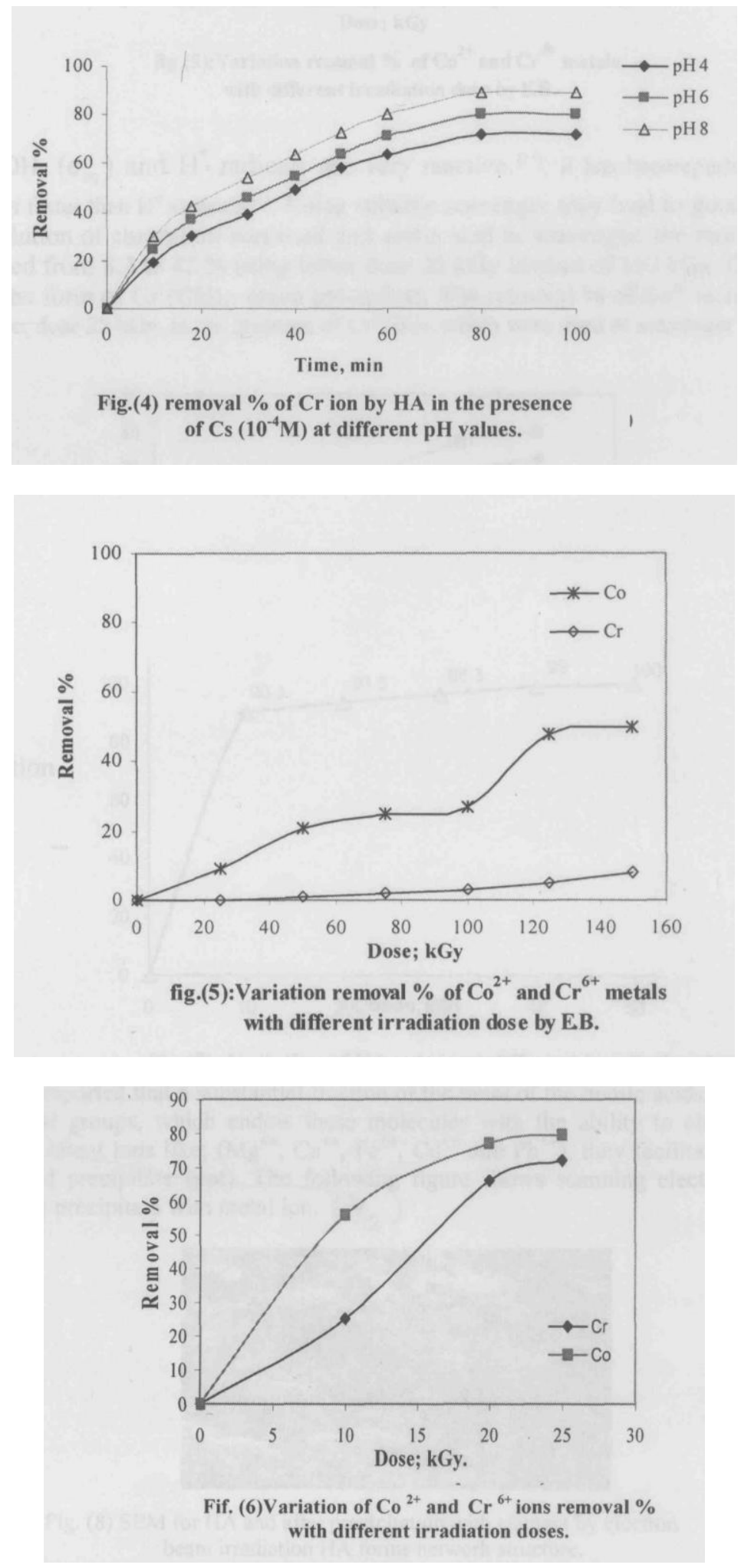

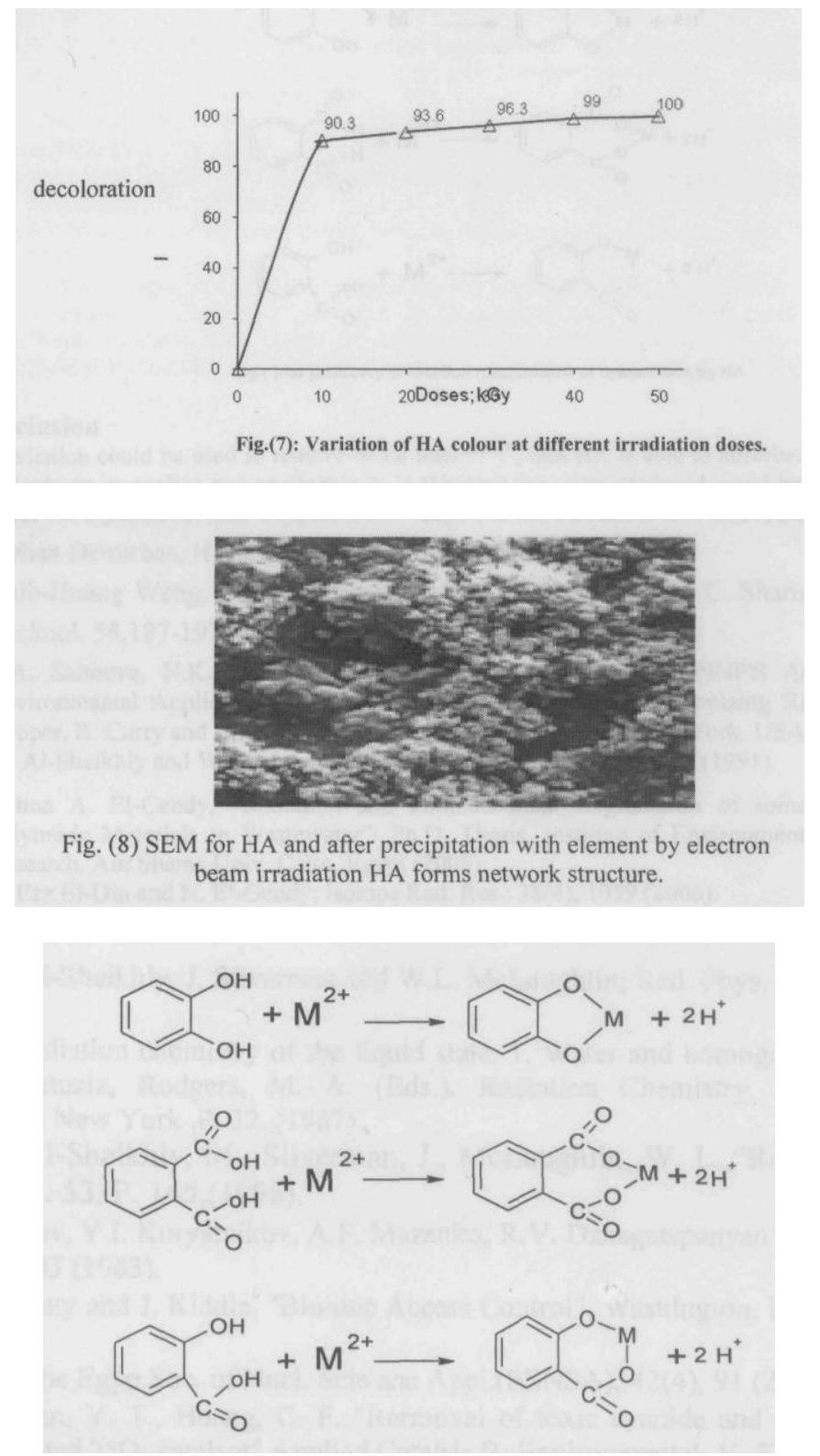

Figure 9: the possibility of reaction mechanisms of uptake ions by HA 
Military Technical College

Kobry El-Kobbah, Cairo, Egypt

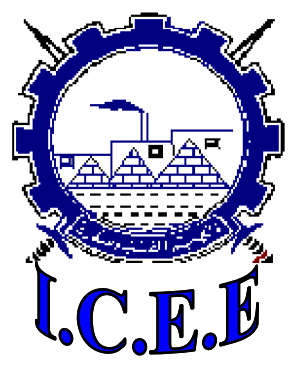

$5^{\text {th }}$ International Conference on

Chemical \& Environmental Engineering 25 - 27 May, 2010. 